\title{
Philosophiques
}

\section{Claude Piché, Kant et ses épigones. Le jugement critique en appel , Paris, Librairie philosophique Vrin (coll. " Bibliothèque d'histoire de la philosophie »), 1995, 248 p.}

\section{Claude Veillette}

Volume 26, numéro 2, automne 1999

La critique de la raison en Europe centrale

URI : https://id.erudit.org/iderudit/004995ar

DOI : https://doi.org/10.7202/004995ar

Aller au sommaire du numéro

Éditeur(s)

Société de philosophie du Québec

ISSN

0316-2923 (imprimé)

1492-1391 (numérique)

Découvrir la revue

Citer ce compte rendu

Veillette, C. (1999). Compte rendu de [Claude Piché, Kant et ses épigones. Le jugement critique en appel, Paris, Librairie philosophique Vrin (coll. "Bibliothèque d'histoire de la philosophie "), 1995, 248 p.] Philosophiques, 26(2), 392-395. https://doi.org/10.7202/004995ar d'utilisation que vous pouvez consulter en ligne.

https://apropos.erudit.org/fr/usagers/politique-dutilisation/ 


\section{Comptes rendus}

Claude Piché, Kant et ses épigones. Le jugement critique en appel. Paris, Librairie philosophique Vrin (coll. « Bibliothèque d'histoire de la philosophie »), 1995, 248 p.

Est-ce une coïncidence si le titre du livre de C. Pichéfait écho à celui d'O tto Liebmann intitulé Kant und die E pigonen publié en 1865? Q uelle que soit la réponse, il n'en demeure pas moins que l'auteur du présent ouvrage nous convie, tout comme ce néokantien de la première heure, à une certaine forme de retour à l'ensemble de l'entreprise critique de Kant. L'intention de C. Piché dans cet ouvrage consiste en effet à montrer combien le thème de la faculté de juger qu'abrite le criticisme offre un point d'ancrage théorique solide pour aborder la discussion contemporaine entourant le problème de la fondation du discours philosophique. $0 \mathrm{n}$ peut repérer dans l'ouvrage trois moments par lesquelsl'enjeu de cette problématique est développé. Dans un premier temps, l'auteur pose les points d'appui qui permettent une « relecture » de la Critique de la raison pure à partir de la faculté de juger définie comme l'instance critique par excellence. Dans un deuxième temps, il expose le sort réservé à cette pièce maîtresse de la Critique qu'est la faculté de juger chez les successeurs immédiats de Kant, en particulier R einhold et Fichte. En dernier lieu, I'auteur se propose d'examiner combien l'héritage de la philosophie néo-kantienne a orienté, dans une certaine mesure, la manière de poser le débat philosophique contemporain, notamment en Allemagne, sur le rôle et la tâche de la philosophie à l'ère post-métaphysique. À cet effet, la philosophie de $\mathrm{H}$ abermas est considérée par l'auteur comme un témoignage éloquent de cette situation.

Le corps del'ouvrage se divise en quatre chapitres dont nous donnerons un bref aperçu dans les lignes qui suivent. Le premier chapitre (p. 13-72) se penche sur le problème de l'autonomie de la liberté théorique chez Kant. II s'agit d'examiner la question de la concordance des concepts purs de l'entendement avec les objets de l'expérience de façon à montrer que la solution à cette question est inséparable du statut que confère la C ritique de 1781 à la faculté transcendantale de juger. Le thème de la concordance avec l'objet pose en effet au jugement la difficulté de savoir comment l'attribution d'un prédicat pur à un objet est possible. $0 r$, comme il en va ici du sens de la fameuse Révolution copernicienne de Kant, la thèse de C. Piché est donc d'affirmer que l'élaboration d'une théorie du jugement est au cœur de la Critique de la raison pure. L'originalité del'auteur sera de montrer que si la faculté de juger ne se laisse définir que par les termes del'A nalytique, en tant que logique de la vérité, cette faculté est par avance condamnée à entériner ce qui, dans le schématisme de l'entendement, assure la médiation entre le phénomène et les catégo ries de l'entendement. II ne peut y avoir de faculté de juger autonome qu'en autant qu'il y a possibilité de méprise et d'erreur. C equi conduit C. Piché à postuler l'idée que la faculté de juger, loin de se restreindreà l'usage auquel I'A nalytique semble l'avoir confinée, traverse de part en part I'ensemble de la Logiquetranscendantale, en tant que logique de l'apparence. Et c'est en suivant cette hypothèse que l'auteur est amené à parler d'un second schématisme, d'un « schématisme de la raison » (p. 56). Le résultat de cette enquête sera d'indiquer que les mauvais usages de la raison ne sont nullement le fait de cette faculté à laquelle 
nous devons les I dées de l'Âme, du M onde et de Dieu, mais bien celui de la faculté de juger elle-même. Et c'est à ce titre que le jugement peut être pensé « comme le nerf d'une liberté théorique qui oscille de manière essentielle entre vérité et fausseté » (p. 55). En somme, ce n'est qu'à partir du moment où la liberté du jugement est assurée qu'une autocritique de la raison prend tout son sens, c'est-à-dire un discours philosophique apte à recenser les connaissances légitimes a priori mais aussi les connaissances philosophiques illégitimes, celles qui sont le fruit de l'illusion dialectique propre à notre disposition métaphysique naturelle.

Dans les deux chapitres suivants, $C$. Piché s'applique à analyser le destin théorique de la faculté de juger et de sa prépondérance sur le sol de la Critique chez deux des principaux représentants de l'esprit systématique propre à la philosophie allemande post-kantienne, à savoir Reinhold (p. 73-128) et Fichte (p. 129-189). Il s'agit ici de rendre compte des motifs qui ont amené ces successeurs de la philosophiekantienne à mettre au rancart la faculté de juger pour les besoins tant d'une « Philosophie élémentaire » que d'une «Doctrine de la Science ». Comme le rappelle l'auteur, la pierre angulaire de la philosophie de R einhold est de doter la philosophie d'un principe qui tienne lieu de fondement unique et absolu. Cen'est pas que R einhold estime que la Critique de la raison pure soit dénuée de fondement, mais il juge que ce qui se donne comme fondement chez Kant sous le terme d'expérience est tout simplement insuffisant. Le concept d'expérience est beaucoup trop contingent et chargé de « présuppositions » pour servir de fondation ultime au discours spéculatif (p. 79). À ce principe, Reinhold substitue celui de conscience. À première vue, ce principe semblebel et bien éviter maints écueils rencontrés avec le principe kantien dela possibilité de l'expérience : il se donne dans un rapport immédiat à la conscience elle-même. L'évidence de cette immédiateté permet ce faisant à R einhold de faire l'économie de l'épineux problème posé par la déduction transcendantale tout en étant persuadé d'avoir satisfait aux exigences de nécessité et d'universalité que réclame la démarche philosophique fondationnelle. $\mathrm{M}$ ais le prix à payer de l'orientation systématique de la philosophie reinholdienne est él evé : le sujet connaissant court le risque de « quitter le terrain de la finitude » (p. 109), oubliant ainsi les conditions d'un rapport dynamique avec le monde.

Le troisième chapitre poursuit la problématique du système doctrinal de la philosophie amorcée au chapitre précédent mais, cette fois, par le biais de l'approche fichtéenne. Tout comme Reinhold, Fichte est à la recherche pour toute la démarche spéculative d'un premier principe. R eprenant le jugement que R einhold adressait à Kant au sujet du caractère incomplet de la fondation de la Critique, Fichte l'étend maintenant à l'entreprise reinholdienne. II reproche notamment à Reinhold de s'être trop livré à l'examen logique des énoncés de la « Philosophie première », occultant ainsi la question de savoir comment se fait l'accès à l'immédiateté de l'évidence qui échoit à l'apriorité du discours philosophique. Se réclamant de la méthode intuitive de la mathématique, l'auteur de la future Wissenschaftslehre trouve en 1793 la clé de la solution dans le principe d'intuition intellectuelle. O utre les précieux détails qu'expose $C$. Pichéau sujet du rapport entre Fichte, R einhold et $K$ ant au sujet de cette notion d'intuition intellectuelle, la section qui traite de la célèbre thèse du primat de la philosophie pratique attire particulièrement notre attention (p. 144-157). Comme le mentionne l'auteur, c'est par la référence explicite à la raison morale que la vérité du système démontrée par l'intuition intellectuelle trouve créance. Autrement dit, seule la croyance en notre destination morale peut assurer la vérité de l'évidence théo- 
rique de l'intuition intellectuelle. En effet, si tout est « dans le moi et par le moi », il s'ensuit que c'est du côté du sujet et non de celui de l'objet que la vérité du système se réalise. O r ce qui, dans le sujet, se dévoile de manière immédiate et spontanée sans aucune médiation, c'est précisément la loi morale. Considérant que seule la loi morale dans le sujet peut obliger le moi à se reconnaître comme spontanéité première, Fichte est amené à envisager l'auto-activité à l'œuvre dans l'intuition intellectuelle comme une spontanéité pratique. En somme, le système spéculatif de Fichte n'accède à la vérité qu'à partir du moment où il est démontré que la « capacité réelle d'agir » que confère la loi morale à la volonté, et par laquelle le sujet se découvre comme spontanéité réelle, est celle-là même qui est à l'œuvre dans l'auto-activité propre à l'intuition intellectuelle. $M$ ais cette façon d'assurer la vérité du discours philosophique par la thèse du primat de la raison pratique se répercute forcément sur la manière de concevoir le rôle de la faculté de juger dans son rapport avec la connaissance en général. Comme l'expose admirablement C. Piché, l'autonomie de la faculté de juger se voit, chez Fichte, grandement amenuisée en raison de la subordination du jugement à I'autorité de la faculté pratique. C'est le sentiment du devoir qui, en tant qu'il est un sentiment immédiat ne souffrant d'aucune médiation, garantit à la connaissance sa certitude. Dans ces conditions, l'acte de porter un jugement perd de ses prérogatives, car il « ne peut mésinterpréter la voix de la conscience morale qui prodigue l'assentiment » (p. 168). Le troisième chapitre se clôt par une très belle analyse de I'histoire de la postérité du fichtéanisme, en portant une attention toute particulière à la reprise des motifs systématiques de la D octrine de la Science par R ickert, qui fut, comme on sait, l'un des principaux représentants du néo-kantisme axiologique de Bade.

Dans le quatrième chapitre (p. 191-245), C. Piché examine certains présupposés théoriques de l'entreprise philosophique de $\mathrm{H}$ abermas. Son intention est de montrer que la théorie habermasienne de la normativité, si chère à son éthique discussionnelle, repose en réalité sur des prémisses empruntées à la philosophie néokantienne. Si par le passé certains interprètes ont déjà soulignél'héritage néo-kantien dans les écrits de $\mathrm{H}$ abermas, notamment dans le programme épistémologique de Connaissance et intérêt (p.192), aucun, à notre avis, n'avait encore autant que C. Piché indiqué l'ampleur de l'influence souterraine exercée par le néo-kantisme dans les tex tes de $\mathrm{H}$ abermas à partir des années quatre-vingt. À cet égard, la lecture que fait $\mathrm{H}$ abermas de la philosophie kantienne est symptomatique du caractère néo-kantien de certains éléments de sa théorie de la communication. Pour étayer sa thèse, $C$. Piché analyse les deux objections qui sont faites à l'endroit du kantisme dans le texte intitulé Philosophie als Platzhalter und Interpret (1981). H abermas reproche d'abord à Kant son « fondationnalisme » qui se reconnaît par la fonction d'assignation qui est dévolue à la philosophie à l'endroit des sciences particulières. II reproche également à Kant d'avoir étendu la juridiction de la philosophie « à l'ensemble du champ de la culture moderne » (p. 194). Cette interprétation de Kant est, aux yeux de C. Piché, tout simplement tendancieuse. Sans entrer dans les détails de l'argumentation de I'auteur, mentionnons simplement que cette méprise est attribuable, selon C. Piché, au fait que $\mathrm{H}$ abermas reprend directement les arguments que R orty, dans Philosophy and the M irror of N ature (1979), avance contre Kant. O r, comme les arguments de Rorty "sont eux-mêmes fondés sur une lecture ouvertement néo-kantienne de Kant », il devient facile en ce cas de localiser la source du désaccord entre $\mathrm{H}$ abermas et $\mathrm{K}$ ant (p. 207). O $\mathrm{n}$ pourrait toutefois penser que ce texte, dans lequel $\mathrm{H}$ abermas 
souscrit au néo-kantisme, est aussi le moment où il y renonce. Car, de fait, ce texte joue un rôle charnière dans l'œuvre de $\mathrm{H}$ abermas : il annonce le passage du paradigme de la connaissance amorcé dans Connaissance et intérêt au paradigme de la communication dével oppé dans Théorie de l'agir communicationnel. Prenant ses distances vis-à-vis d'une théorie de la société menée sous la juridiction de l'épistémologie au profit d'une pragmatique formelle du langage, il est effectivement tentant de penser que la théorie de la communication de $\mathrm{H}$ abermas tourne le dos au néo-kantisme. $M$ ais, comme le démontre $C$. Piché, les choses ne sont pas aussi simples, car avec la pragmatique du langage surgit le thème de « prétentions à la validité ». O r, le thème général de validité est lui-même hérité du néo-kantisme. Ce qui fera dire à $C$. Piché que $\mathrm{H}$ abermas, au début des années quatre-vingt, passe « du néo-kantisme logiciste ( $M$ arbourg) au néo-kantisme axiologique (pays de Bade) » (p. 216).

L'ouvrage de $C$. Piché met en valeur d'une manière fort intéressante combien il est difficile, à notre ère post-métaphysique, d'accéder à la figure originale de la philosophie kantienne. Ce livre a le mérite de participer à la fois à ce qu'il est convenu d'appeler la Kantforschung et à la discussion contemporaine concernant la question de la fondation du discours philosophique. Soulignons enfin la qualité de l'ouvrage, la richesse de l'information et la rigueur dans le traitement de la problématique. 